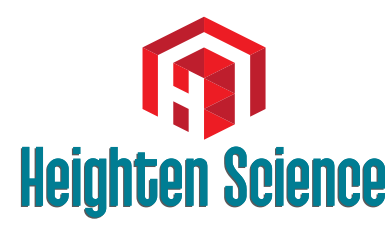

P U B L I C A T I O N S Corporation

\title{
Anesthetic considerations for endovascular repair of ruptured abdominal aortic aneurysms
}

\author{
KH Kevin Luk* and Koichiro Nandate ${ }^{2}$ \\ ${ }^{1}$ Assistant Professor, Divisions of Critical Care Medicine and Neuroanesthesiology \& \\ Perioperative Neurosciences, Department of Anesthesiology \& Pain Medicine, Harborview \\ Medical Center, University of Washington School of Medicine, USA \\ ${ }^{2}$ Associate Professor, Department of Anesthesiology \& Pain Medicine, Harborview Medical \\ Center, University of Washington School of Medicine
}

*Address for Correspondence: KH Kevin Luk, M.D., M.S., Assistant Professor, Department of Anesthesiology \& Pain Medicine, Harborview Medical Center, $3259^{\text {th }}$ Ave, Box 359724, Seattle, WA 98104-9724, USA, Tel: +1 (617) 407-2881; Email: kluk@uw.edu

\section{Submitted: 26 July 2018}

Approved: 10 September 2018

Published: 11 September 2018

Copyright: @ 2018 Kevin Luk KH, et al. This is an open access article distributed under the Creative Commons Attribution License, which permits unrestricted use, distribution, and reproduction in any medium, provided the original work is properly cited

Check for updates

\section{Abstract}

Ruptured abdominal aortic aneurysm (rAAA) carries high morbidity and mortality. Advances in endovascular techniques in the last two decades allow for minimally invasive approach for repair of these aneurysms. A succinct but comprehensive pre-operative is essential for delivery of a safe anesthetic for the patient with rAAA. Placement of proximal occlusion balloon in the descending aorta using the rapid control technique can be life-saving. Endovascular aortic repair (EVAR) can be performed under monitored anesthesia care using local anesthetic and IV sedation, and with fewer invasive lines. However, rapid conversion to general endotracheal anesthesia should be expected. Anesthesiologists should be familiar with the hemodynamic management of rAAA and be ready to provide resuscitation to correct for anemia, coagulopathy, and acidemia. In addition, the anesthesiologist should be aware of the common complications related to EVAR, including abdominal compartment syndrome, distal ischemia, and local vessel injury.

\section{Introduction}

Even for experienced anesthesiologists, caring for a patient with a ruptured abdominal aortic aneurysm (rAAA) is rather challenging and stressful. It carries significant morbidity and mortality with in-hospital morality rate approaching $50 \%$ even in patients who had undergone successful open repair [1]. Shortly after the introduction of endovascular aneurysm repair (EVAR), it has been shown to reduce operative mortality substantially. Approximately half of the rAAA cases are considered favorable for endovascular repair [2,3]. For those who had undergone EVAR for rAAA, the in-hospital mortality had been reduced to $20-30 \%$ [4,5]. Due to the minimally invasive nature of the EVAR technique, for elective EVAR cases, central venous catheter are not necessary, and arterial catheterization can often be omitted. Monitored anesthesia care with local anesthetic infiltration and IV sedation, as well as regional (e.g. lumbar subarachnoid block) technique are gaining popularity for elective EVARs over general anesthesia. Although more and more EVAR for rAAA were done under monitored anesthesia care with local anesthetic and IV sedation, the optimal anesthetic technique for the management of EVAR for rAAA remains unclear.

\section{Discussion}

\section{Preoperative evaluation / preparation}

Although rAAA is considered a life-threatening condition and requires immediate attention to decrease the risk of end organ perfusion deficit and restore circulatory volume, it is prudent for the anesthesia team to gather as much information from 
the patient as possible prior to the beginning of the case. Ideally, the patient should be interviewed and evaluated by the anesthesia in the preoperative holding area or the emergency department. However, most centers have now adopted policy of bypassing the emergency department for patient transferred from an outside facility with a diagnosis of rAAA. These patients are routed to the operating room directly. Therefore, only a brief interview might be possible. The interview should focus on pertinent past medical / surgical history, allergies, and medications. Vascular access, medications administered, and hemodynamic status should be reviewed. A brief airway examination should be performed.

After interviewing and examining the patient, it is important to discuss with the surgical team regarding the surgical plan and whether open repair is feasible as a backup if the attempt at endovascular repair failed. Prior to the repair, the surgeon may opt to employ the Resuscitative Endovascular Balloon Occlusion of the Aorta (REBOA) technique. This is achieved by placement of an aortic occlusion via a transfemoral artery approach and deploying the balloon in the supra-celiac position as a rapid vascular control technique. This serves to stabilize the patient's hemodynamic status and maintain adequate end-organ perfusion. Previous reports have shown that such rapid control technique decreased mortality in unstable rAAA patients [6,7]. The occlusion balloon is deployed in the segment of the descending aorta that is proximal to the celiac axis takeoff. Therefore, prolonged inflation can result in hepatic and mesenteric ischemia, which can worsen coagulopathy and metabolic acidosis. Ideally, the balloon inflation time should be limited to less than 30 minutes. In addition, anesthesiologists should be aware that fluoroscopy is required for placement of the catheter, which can result in patient's both arms being tucked, which can render placement of radial artery catheter unfeasible.

\section{Coagulopathy \& blood transfusion for rAAA}

Massive hemorrhage in patients with rAAA undergoing either open repair or EVAR has been associated with poor outcome, and coagulopathy is an early complication. Disturbance of fibrinogen level, international normalized ratio (INR), prothrombin Time (PT), activated partial thromboplastin time (APTT) and platelets counts are frequently observed [8-10]. Therefore, it is prudent for the anesthesia team to activate the "Massive Transfusion Protocol" and obtain universal donor blood products from the blood bank. There is insufficient evidence for the optimal strategy for transfusion in rAAA patients. The literature is mostly derived from the trauma population [11].

EVAR is associated with lower transfusion volumes than open aneurysm repair. However, recent investigation reports that $55 \%$ of EVAR patients requiring massive transfusion. During the initial resuscitation phase, we recommend transfusion in a 1:1:1 PLT:RBC:FFP ratio until the hemorrhage is controlled. Compared to open procedures, it is more difficult to evaluate coagulopathy by direct surgical field observation during EVAR. Therefore, emergency hemorrhage panels (EHP, includes: hematocrit, INR, platelet count, and fibrinogen level) or thromboelastography (TEG) may be very helpful to diagnose coagulopathy and tailor the transfusion strategy after initial resuscitation. At our institution, EHP is checked after the administration of each massive transfusion pack, followed by hourly monitoring until hemostasis is achieved.

\section{Acid-base disturbance}

Most patients presenting with rAAA are hypovolemic due to massive hemorrhage. This results in a gap metabolic acidosis with lactemia. The key therapy is to restore circulatory volume and oxygen carrying capacity using the transfusion strategy stated in the transfusion section. Sodium bicarbonate should be reserved for patients with severe metabolic acidosis with hemodynamic instability or life threatening hyperkalemia resulting in arrhythmias. Administration of sodium bicarbonate 
will result in increase in $\mathrm{P}_{\mathrm{a}} \mathrm{CO}_{2}$ as bicarbonate is being converted to carbon dioxide. This can result in an increase in minute ventilation (MV). For patients that are not undergoing GA and mechanically ventilated, the increase in MV might not be well tolerated. Tromethamine (THAM) has been advocated as an alternative to bicarbonate for treatment of metabolic acidosis [12]. The reaction results in elimination of $\mathrm{CO}_{2}$, but the elimination of THAM requires intact renal function.

\section{Anesthetic technique}

Open aneurysm repair necessitates the use of general endotracheal anesthesia (GA). However, the minimally-invasive nature of EVAR allows for monitored anesthesia care (MAC) using local anesthetics and IV sedatives. Regional techniques have also been employed but are generally limited by patient cooperation and heparinization during the procedure. Previous studies have demonstrated that MAC resulted in fewer pulmonary complications, shorter ICU and hospital length of stay for elective EVAR cases [13]. In addition a meta-analysis showed that general anesthesia is an independent risk for death after EVAR [14]. In current literature, there are two case reports of regional or local anesthesia with IV sedation for the endovascular treatment of Raaa $[15,16]$. However, there are no randomized, controlled trials to determine if the anesthetic technique results in difference in patient outcomes.

\section{Choice of sedatives \& analgesics}

Midazolam, fentanyl, propofol, ketamine, and dexmedetomidine have been used either alone or in combination as the sedative / analgesic agent(s) for EVAR. However, there is no consensus regarding the optimal sedative for EVAR. The patient should be able to respond to commands to hold their respiration for the injection of contrast and deployment of the stent-graft. With our experience, dexmedetomidine has the most desirable properties to satisfy those specific requirements of EVAR. A previous retrospective analysis has reported that dexmedetomidine is useful alternative to general anesthesia for EVAR in terms of anesthesia and surgical time, pain score, pain medication use and hemodynamic variance [17]. Dexmedetomidine does not depress respiratory drive so it would not exacerbate pre-existing acidemia in rAAA patients. However, it should be noted that the central alpha- 2 agonism can potentially result in hypotension in a hypovolemic patient.

\section{Considerations for conversion from IV sedation to general anesthesia}

The general indications for conversion from MAC to GA includes:

1. Conversion of EVAR to open aneurysm repair

2. Compromise of oxygenation and ventilation

3. Patient's inability to cooperate or tolerate the procedure under MAC

4. Patient agitation / restlessness

In terms of induction drugs of choice, etomidate and ketamine are preferred over propofol or sodium thiopental because of their lesser negative effect on the cardiovascular system. It is imperative to be careful with airway manipulation since the patient is fully heparinized, so that even minor tissue trauma can result in significant bleeding in the airway. This can obscure the view of the glottis for intubation. At our institution, we employ video laryngoscopy for endotracheal intubation even in cases where difficult airway is not suspected.

In terms of the decision to extubate the patient at the end of the procedure, it largely depends on the initial indication of conversion. It is not always possible for patient to emerge from GA especially the cause of conversion is due to poor mental status, or 
inability to keep up with oxygenation / ventilation. Our general practice is to keep the patient intubated and sedated for at least 24 hours post-operatively in the intensive care unit to monitor for potential complications such as abdominal compartment syndrome, renal failure, coagulopathy or other surgical complications.

\section{Monitoring devices \& invasive lines}

Ideally, an arterial line and at least two large bore peripheral lines should be established before the surgery starts. However, it is very important for the anesthesia team not to delay the deployment of the REBOA balloon for proximal control of the hemorrhage, because the rapid control technique is essential for patient survival. Furthermore, the peripheral pulse might be weak due to hemorrhagic shock, so arterial catheterization can be difficult even under ultrasound guidance. Therefore, it may be appropriate to delay invasive arterial line placement until the blood pressure improves after the surgical team has deployed the REBOA balloon in the proximal descending aorta. Central venous access is optional unless the peripheral access is very poor, and it might be technically challenging due to low intravascular volume. An alternative is a surgeon-placed central venous catheter in the femoral vein, especially if conversion to general anesthesia is anticipated.

\section{Monitoring of anticoagulation}

Systemic heparinization at an initial dose of 80-100 units/kg of unfractionated heparin is necessary for EVAR. The activated clotting time should be monitored and maintained at greater than 250 seconds. The ACT should reassessed hourly and unfractionated heparin redosed as necessary to maintain therapeutic anticoagulation. At the conclusion of the procedure, prior to removal of the access sheath(s), ACT is measured again to determine if reversal of anticoagulation is necessary. At our institution, if the ACT is $>200 \mathrm{~s}, 20-30 \mathrm{mg}$ of protamine is administered to reverse the effect of heparin.

\section{Procedural complications related to EVAR}

EVAR is an effective and less invasive alternative to open surgery, but it is not free of complications. Three perioperative complications of EVAR deserve special attention [18-20].

\section{Abdominal Compartment Syndrome}

Abdominal compartment syndrome (ACS) is a common complication after open aneurysm repair and is thought to be related to third spacing with subsequent increase in intra-abdominal pressure. However, ACS after EVAR is not uncommon, and the mechanism of pathogenesis is different. Patients who developed ACS after EVAR were observed to have received significantly more volume resuscitation intraoperatively compared to EVAR patients without ACS or patients with ACS after open aneurysm repair. It is thought that the bleeding can occur during graft placement, or through an on-going Type II endoleak via inferior mesenteric or lumbar vessels.

\section{Arterial Injury at the Vascular Access Site}

Occasionally, the external iliac or femoral arteries are injured during insertion, and rarely during removal of the delivery sheath. This may cause significant bleeding into the retroperitoneal space, which is not visible in the surgical field. To confirm such injury, emergency arteriogram is necessary. Therefore, attention should be paid to changes in blood pressure before and immediately after the insertion or removal of the delivery sheath. If a significant decrease of BP is observed, the surgical team must be informed and blood samples sent to check hemoglobin and coagulation status. Previous reports suggest that this is a rare, but significant complication that impacts eventual outcomes [19]. 


\section{Early Lower Extremity Ischemia and Arterial Occlusion}

Early lower extremity vascular occlusion is another potential complication following EVAR. It is often not recognized until the surgeons check the pulse in the lower extremities. Therefore, the patient should be kept sedated until the distal peripheral pulses are confirmed. If pulses are weak or absent, the surgeons may need to explore the femoral insertion site under general anesthesia.

\section{Conclusion}

In summary, rAAA carries significant morbidity and mortality in patients typically with multiple comorbidities. Advances in endovascular therapy allows for repair of the aneurysm in a minimally-invasive fashion. Anesthesiologists should be familiar with the hemodynamic management and blood product resuscitation strategy in these patients. In addition, decision should be made for MAC vs GA with the ability to convert in an expeditious but safe manner. The anesthesiologist should also be aware of the potential complications related to EVAR and be able to assist the surgeon with the management if they arise.

\section{References}

1. Ellard L, Djaiani G. Anesthesia for vascular emergencies. Anaesthesia. 2013; 68 (Suppl 1): 72-83. Ref.: https://goo.gl/4YV6tY

2. Slater BJ, Harris EJ, Lee JT. Anatomic suitability of ruptured abdominal aortic aneurysms for endovascular repair. Ann Vasc Surg. 2008; 22: 716-722. Ref.: https://goo.gl/Gc85Rz

3. Hoornweg LL, Wisselink W, Vahl A, Balm R. Amsterdam Acute Aneurysm Trial Collaborators. The Amsterdam acute aneurysm trial: suitability and application rate for endovascular repair of ruptured abdominal aortic aneurysms. Eur J Vasc Endovasc Surg. 2007; 33: 679-683. Ref.: https://goo.gl/3XzHtz

4. Bown MJ, Sutton AJ, Bell PR, Sayers RD. A meta-analysis of 50 years ruptured abdominal aortic aneurysm repair. Br J Surg. 2002; 89: 714-730. Ref.: https://goo.gl/FrBmiJ

5. Mastracci TM, Garrido-Olivares L, Cina CS, Clase CM. Endovascular repair of ruptured abdominal aortic aneurysms: systemic review and meta-analysis. J Vasc Surg. 2008; 47: 214-221. Ref.: https://goo.gl/WPtYfh

6. Phillipsen TE, Hendriks JM, Lauwers P, Voormolen M, d'Archambeau O, et al. The use of rapid endovascular balloon occlusion in unstable patients with ruptured abdominal aortic aneurysm. Innovation. 2009; 4: 74-79. Ref.: https://goo.gl/PV5Aew

7. Raux M, Marzelle J, Kobeiter H, Dhonneur G, Allaire E, et al. Endovascular balloon occlusion is associated with reduced intraoperative mortality of unstable patients with ruptured abdominal aortic aneurysm but fails to improve other outcomes. J Vasc Surg. 2015; 61: 304-308. Ref.: https://goo.gl/RgLjx5

8. Montan C, Hammar U, Wikman A, Berlin E, Malmstedt, Holst J, et al. Massive blood transfusion in patients with ruptured abdominal aortic aneurysm. Eur J Vasc Endovasc Surg. 2016; 52: 593-603. Ref.: https://goo.gl/yiB3Gi

9. Montan C, Joansson F, Hedin U, Wahlgren CM. Preoperative hypofibrinogenemia is associated with increased intraoperative bleeding ruptured abdominal aortic aneurysms. Thromb Res. 2014; 135 : 443-448. Ref.: https://goo.gl/nN46u5

10. Fransson $\mathrm{M}$, Rydningen $\mathrm{H}$, Henriksson AE. Early coagulopathy in patients with ruptured abdominal aortic aneurysm. Clin Appl Thromb Hemost. 2012; 18: 96-99. Ref.: https://goo.gl/akBujq

11. Holcomb JB, Tilley BC, Baraniuk S, Fox EE, Wade CE, et al. Transfusion of Plasma, Platelets, and Red Blood Cells in a 1:1:1 vs a 1:1:2 Ratio and Mortality in Patients With Severe Trauma: The PROPPR Randomized Clinical Trial. JAMA. 2015; 313: 471-482. Ref.: https://goo.gl/94HL8s

12. Hoste EA1, Colpaert K, Vanholder RC, Lameire NH, De Waele JJ. et al. Sodium bicarbonate versus THAM in ICU patients with mild metabolic acidosis. J Nephrol. 2005; 18: 303-307. Ref.: https://goo.gl/G7jEkN

13. Edwards MS, Andrews JS, Edwards AF, Ghanami RJ, Corriere MA, et al. Results of endovascular aortic aneurysm repair with general, regional and local/monitored anesthesia care in the American College of Surgeons national Surgical Quality Improvement Program data-base. J Vasc Surgery. 2011; 54: 1273-1282. Ref.: https://goo.gl/85dgXN 
14. Walschot LH, Laheij RJ, Verbeek AL. Outcome after endovascular abdominal aortic aneurysm repair: meta-analysis. J Endovascular Ther. 2002; 9: 82-89. Ref.: https://goo.gl/vqUBWW

15. Smith TA, Ramirez D. Awake, percutaneous repair of ruptured abdominal aortic aneurysm. Ochsner J. 2013; 13: 248-251. Ref.: https://goo.gl/XF4mGa

16. Tay JW, Leong YP. Ruptured abdominal aortic aneurysm treated by endovascular aneurysm repair under local anesthesia. Md J Malaysia. 2012; 67: 111-112. Ref.:@ https://goo.gl/qLH19S

17. Brown BJ, Zakhary S, Rogers L, Ellis-Stoll C, Gable D, et al. Use of dexmedetomidine versus general anesthesia for endovascular repair of abdominal aortic aneurysms. Proc (Bayl Univ Cent). 2006; 19 : 213-215. Ref.: https://goo.gl/jXjMws

18. Maleux G, Koolen M, Heye S. Complications after endovascular aneurysm repair. Semin Intervent Radiol. 2009; 26: 3-9. Ref.: https://goo.gl/zsUdmK

19. Shah SK, Parodi FE, Eagleton MJ, Bena JF, Clair DG. Iliac injury during abdominal and thoracic endovascular intervention. J Vasc Surg. 2016; 64: 726-730. Ref.: https://goo.gl/2FnLgN

20. Rubenstein C, Bietz G, Davenport DL, Winkler M, Endean ED. Abdominal compartment syndrome associated with endovascular and open repair of ruptured abdominal aortic aneurysms. J Vasc Surg. 2015; 61: 648-654. Ref.: https://goo.gl/F824pD 